\title{
Activities of Traditional Birth Attendants in Okpatu, Udi Local Government Area Enugu,Nigeria
}

\author{
Odikpo Linda.C ${ }^{1,}$ Akpan, Mfon .G. ${ }^{1}$,Kanikwu, Nwamaka ${ }^{1}$, Irodi.c. ${ }^{2}$ \\ ${ }^{1}$ Department Of Nursing Sciences, University Of Nigeria, Enugu, Nigeria . \\ ${ }^{2}$ Department Of Nursing Sciences IgbenedionUniversity Okada,Benin, Nigeria .
}

\begin{abstract}
This study was conducted to investigate the activities of Traditional birth attendants (TBAs) in Okpau community, Udi L.G.A of Enugu state. The respondents comprising of 10 TBAs that were resident within the 8 villages visited, constituted the study sample. Snowballing sampling technique was used. In-depth interview guide was the tool used for data collection and data was analyzed using thematically. The results reveal that TBAs generally provide some antenatal services though majority do not understand the meaning of the results, majority of TBAs use and understand signs of labour, the TBAs do not consider post -partum care as important, the TBAs knowledge of detecting risk factors is poor and majority of the TBAs make use of herbs and/or prayers to manage their clients. In conclusion, it was noted that TBAs have been unsuccessful in handling obstetric complications, but have contributed to successful maternal, neonatal, and child health interventions. Therefore, it was recommended that more and well staffed health centres and hospitals should be cited in okpatu to increase accessibilyto care especially at night, there should be proper training of TBAs on all round care and management of patints.
\end{abstract}

Keywords: Activities, Enugu, Okpatu, Traditional Birth Attendants.

\section{Introduction}

Each year around four million new born babies die in the first week of life, worldwide, and an estimated 529,000 mothers die due to pregnancy-related causes. In low and middle-income countries many deliveries still occur at home and without the assistance of trained attendants but by traditional birth attendants (TBAs). This has generated serious concern, since women who develop life-threatening complications during pregnancy and delivery require appropriate and accessible care ${ }^{[1][2]}$.

A TBA has been defined by United Nations as a person who assists mothers during childbirth and initially acquired her skills by delivering babies herself or through apprenticeship to other TBAs. TBAs presently deliver the majority of women in Nigeria as in other developing countries. However, some of the TBAs do not feel free to perform their duties because of the conflicts they encounter with orthodox medical personnel and the fear of non-registration by the establishment. The doctors and nurses believe that the TBAs are neither trained, skilled nor knowledgeable about the delivery of babies whereas the TBAs on the other hand hold on to the "authoritative knowledge" as they are the ones that deliver majority of children of people in areas where there are public and private modern hospitals and health facilities, and even of people who are too poor to pay the fees in hospitals. The practice of TBAs in the rural context cannot be underrated ${ }^{[2]}$. It is estimated that between 60 and $80 \%$ of all deliveries in the developing countries occur outside modern health care facilities, with a significant proportion of this attended to by TBAs.

Millennium Development Goal (MDG) 4 aims to reduce mortality of children, aged less than five years, by two-thirds between 1990 and 2015. MDG 5 aims to reduce maternal mortality by three quarters during this timeframe. Policy regarding the best strategies to meet these goals, however, swings between community-based care and facility-based care, while safe motherhood and child-survival advocates compete for limited resources .

According to the Nigeria Demographic and Health Survey 2008, between 2003 and 2008, only 46\% of women living in rural areas received antenatal care from a skilled provider (ie, doctor, nurse/midwife, auxiliary nurse/midwife), mothers who cannot access these services are left to make do with "alternatives" such as TBA services. Yet efforts to formalize the role of TBAs in maternal and neonatal health programs have had limited success. TBAs continued attendance at home deliveries suggests, however, their potential in influencing maternal and neonatal outcomes. More so, as practicing mid-wives, it was a very common practice to have very complicated cases, e.g, post- partum hemorrhage, ruptured uterus, intra uterine deaths and others being rushed from TBAs for possible remedies in the hospital. Thus, With the high maternal mortality rate, rising prevalence of HIV/AIDS, insufficient number of skilled health care providers and high level of utilization of TBAs, , there is an urgent need for research of this kind to assess the activities of TBAs in Okpatu, Udi Local Government Area of Enugu State.

In a study by ${ }^{[3]}$ to assess the activities of TBAs in Akwalbom State, the services provided by the TBAs ranged from ante-natal care, child delivery, treatment of infertility, management of threatened abortion, and 
circumcision of babies. Preparations used in the treatment of cord stump included menthylated spirit, herbal preparations, dry heated sand and engine oil. Some of the medications also used were animal dung, flies, scarification marks, and cow urine. Methods of risk assessment during ante-natal care, management of delivery complication, record- keeping among TBAs were found to be poor. Infection prevention methods used were also found to be very poor. According to ${ }^{[5]}$, these harmful practices result to some complications.

The objective of this study is to assess the activities of TBAs in Okpatu, Udi Local Government Area of Enugu State. Although, there had been similar studies done in both developed and developing countries; including Nigeria, there has not been any empirical study up to the time of this study that the researchers are aware of, on Activities Of Traditional Birth Attendants in Udi Local Government Area. By exploring this gap, this study seeks to clearly understand how Traditional Birth Attendants care for women of this locality from antenatal, delivery, and post -partum, as this knowledge will help broaden our view to the important role these rural women play in health care delivery, and it will serve as empirical evidence to enhance better training of traditional birth attendants, aiming at reducing fetal and maternal mortality. Therefore, the question is, what are the activities of TBAs in Udi LGA?

\subsection{Research Design}

\section{Methodology}

It is a qualitative phenomenological type of study. This type of study is important in laying foundation for later research. It is most appropriate method of analysis when in-depth interview is used for data collection.

\subsection{Description of Study Area}

The study was conducted at Okpatu community of Udi Local Government Area, Enugu State. It is a rural community that is made of twelve (12) villages amongst which eight (8) villages namely: Umuleme, Obiagu, Amaegbu, Amazi, Umuchime, Amaen, Eziama, and AmaguUmene were used in the study.

\subsection{Target Population}

The target population for the study population comprised only traditional birth attendants resident in Udi Local Government Area

\subsection{Sampling Technique and Sample Size}

A Snowball sampling technique was used in which the TBAs were traced to their homes through the help offocal persons who were mostly TBAs in each community visited. A total of ten (10) practising TBAs were located and used for this study

\subsubsection{Inclusion criteria:}

1. TBAs must be a mother

2. Must have been practicing for more than 10 years

3. Must be a resident in the Community

\subsubsection{Selection of interviewees}

Participants for each interview were selected based on the inclusion criteria. The selection of villages was conducted after consultations between the researchers and the health officer in-charge of Okpatu health centres A focal person was appointed from each village and date of next meeting communicated to them. Indepth interview was used to gather information from the participants. In each community visited, the TBAs were located in their homes through the help of a focal person. The interviews were conducted in the interviewee's house using the guide and all the interviews were audio recorded.

There were (4) two researchers for this work, two of the researchers served as interviewers, while the others played the role of a note takers. Each session was tape recorded. The content of the taped version was transcribed and analysed. The analysis was done by categorizing the collected data using research objectives.

The next category was done by examining the relationship among different categories. Each interview session take about 45 minutes. We included TBAs who are both indigenes and non-indigenes residing at the different communities. The Community leaders were informed of the study objectives and participant inclusion criteria.

\subsection{Instrument for Data Collection}

The research instrument was an interview guide which consisted of twelve (12) open ended questions. The questions were arranged in two (2) sections, questions in section " $A$ " aimed at generating participant's demographic data while questions in section "B" were used to elicit the activities of TBAs. 


\subsection{Data Analysis}

Data were analysed using the following sequence:

Transcribing and sorting

Organising and ordering

Repeatedly going through the collected data

Coding or labelling of different sections

Development of themes, and analysis done thematically.

At the end of the above steps, the following themes were generated for this analysis:

- Services provided by TBAs during pregnancy.

- Services provided by TBAs during labour.

- Services provided by TBAs during puerperium.

- Detection of risk factors by TBAs.

- Management of complications by TBAs.

- Use of herbs/prayers as remedy by TBAs

- When and where referral is done.

\subsection{Services provided during pregnancy}

\section{Findings of the Study}

Some of the TBAs chose Mondays as the day that they would stay at home to attend to any pregnant woman. On this day, they claim to be checking their haemoglobin using the colour chart, but when one of the TBAs was asked to explain how she does this, she brought out the chart and said "I go just compare the colour with ither their palm or their eyes, that is whether red like this colour." They would also check their blood pressure, a though most of them do not know the normal range. For instance, a participant responded when asked to tell uswhat she would regard as being too low or too high. "from that kind 200 is high but when it is 100 is low." And another said, "from 170/100 is high." All the participants accepted to be doing abdominal palpation to see if the child is lying well' but none could really explain how a normal fetal heart sounds. For instance, a participant sary said "I here well 36 beats for 1hour." None of the TBAs kept correct record of their clients, as none was able to produce any record when they were asked. Generally, none of the TBAs ran an organised antenatal system, they will just advise or treat patients who come to them with problems in pregnancy

\subsection{Services provided during labour.}

Majority of the TBAs used clinical features like, pains, show and cervical dilatation to diagnose labour and imminent delivery. They however, do not understand how to measure cervical dilatation in centimeters. This was inferred from the response of one of the respondents when she was asked how she knows when a woman is in labour, she said "I have theatre now! I use my finger to assess VE, if it is tight she is not ready, but something like catarrh will come out for like a week, after that it will come with blood, then is ready." Another observation was that almost all of them use oxytocic drip to either augment or accelerate labour. Example, a participant, when asked how she manages her clients in labour said; "I normally put one bottle of oxytocin into drip and put them till they deliver." For full dilatation of cervix, the TBAs use spontaneous rupture of membranes, appearance of the fetal head and client's complain of the urge to go to toilet. Two of the TBAs said they give episiotomy to primi-gravidae with new razor blades and suture with catgut but they were not able to suture in layers. This was inferred from a participant's response when asked how she sutures big cuts; she said "I go put one or two threads, you know say edey pain them." They all responded to be using a bottle or two of ergometrine injection after delivering of placenta to control bleeding but if it continues, paw-paw leave is squeezed to have a spoonful of the extract and a pinch of salt is added for the woman to drink. The participant said; "it stops bleeding within 5-10 minutes."

\subsection{Services provided during puerperium.}

For the services during puerperium, a TBA said; "They go home after like three hours after delivery." Another said; "I let them go home the next day' I dey tell them if they get problem, they fit come back."

\subsection{Detection of risk factors}

Attempts to assess the risk factors were made by TBAs by assessing the client's blood pressure, haemoglobin estimation and urinalysis, but they are doing them as routine activities. They lack knowledge to decipher the normal from abnormal as such, cannot recognise imminent risk from these activities. However, some of them still have their quick ways of identifying danger signs. For instance, a TBA was asked if there's a way she could see a client during pregnancy and know that she needs to see a doctor because, she could have problems before, during or after labour. She answered this way "If I see the person having big swollen legs, getting weak- eno get power at all or the blood is not normal, I'll refer to health centre." 


\subsection{Management of complications.}

The normal duration of pregnancy by the TBAs was nine (9) lunar months, but when it gets to ten(10) lunar months, the pregnancy was considered to be prolonged. Castor oil is usually given orally and plain water enema to initiate labour. The TBAs consider labour to be prolonged and obstructed if the client has laboured for more than ten(10) hours irrespective of their parity. This was inferred from the response of a TBA when she was asked how long a woman can labour before she is convinced that there is something with the delivery. She responded thus, "I go give them time well well, because each baby whether first or second, get him own style of wakka."

\subsection{Use of herbs/prayers as remedy.}

Most of the TBAs used herbs/prayers for prevention and management of complications. Almost all of them use one form of herb or the other singly or in combination (two or more herbs mixed together). For instance, a TBA when asked how she manages post -partum bleeding said, "paw-paw leaves with salt, I squeeze up to 1 spoonful for the woman to drink, stop bleeding within 5-10 minutes." Another TBA when asked the types of herbs she uses in her practice said," I use bark of Iroko cut into pieces, soak in a bottle of water for 2-3 days, I give to pregnant woman of about 6 (six) months, twice a day to reduce the baby's size when it is too big." At another instance a TBA mention in her local dialect that she uses root of "Agwu-Ilo" as drink for reduction of baby's size while the leaves of "Agwu-oludo" was squeezed and given as drinks for removal of retained placenta. The same TBA said "I dey pray well-well make the leaves work as I dey give."

\subsection{When and to where referral is done.}

It was discovered that most of the TBAs refer their clients to a private clinic of their choice only when they are convinced that they cannot handle the condition, which is strictly discretional. This was inferred from the response of a TBA when she was asked at what point does she refer a client and to where? She replied thus "when I see that the woman is very weak or may be eye begin turn then I fit carry go meet doctor for ninth mile," They do not have specific indications to help them refer clients on time. However, each of them mentioned a particular clinic that she refers their clients to, when they can no more handle the case.

\section{Discussion of Findings}

- TBAs generally provide some antenatal services though majority does not understand the meanings of the result.

- The majority of TBAs use and understand signs of labour.

- The TBAs do not consider post -partum care as important.

- The TBAs knowledge of detecting risk factors is poor.

- Majority of the TBAs make use of herbs and/or prayers to manage their clients.

- Most of the TBAs refer their clients to a private clinic of their choice only when they are through with their manipulation.

In this research, it was discovered that most TBAs provide antenatal services, for example, they check the pregnant women's haemoglobin, blood pressure, carry out palpation and listen to foetal heart sound. This result is in line with studies conducted by, [6], where they identified management and care of patient during pregnancy as one of the expected role of TBAs. This shows the importance of quality care of the women during pregnancy, hence, the TBAs need adequate training to enhance proper observation of patients and accurate interpretation of their findings since most of them do not know or understand the meanings of the result they get during assessment.

However, majority of TBAs do recognize and understand signs of labour. They generally do vaginal examination to monitor the progress of labour. This is in agreement with the , [1], which indicated that one of the expected roles of TBAs is management and provision of care to patients during labour. The need for the training of TBA remains important as most TBAs do not interpret vaginal exam, and cannot detect complications promptly due to wrong interpretations of examination during labour.

The TBAs do not consider post- partum care as important as most of them only tell the patient to come if there is problem. They don't even consider 6weeks postpartum visit as important hence there is need to expose TBAs in- training to some of the landmarks of checking a woman during postpartum period and its importance such as; the checking of uterine involution, changes in lochiae and post-partum observation of the newborn during post -partum visit .

The TBAs knowledge of detecting risk factors is poor hence there is need for adequate orientation and education on things that constitute risk during pregnancy, labour and delivery. This is important as,[1] identified early identification of high risk patients and prompt referral to hospital as one of the expected role of TBAs.

Again, the indiscriminate use of drugs and herbs is also a cause for concern. This is because most 
TBAs use drugs singly or in combination with herbs not knowing their mechanisms of action and correct dosage. This has resulted in the death of some women due to improper administration of these drugs and herbs. This is not to say that TBAs should not make use of drugs, but there is need to understand the active ingredients in these drugs and herbs, their mechanisms of action and the correct dosage to prevent health complications. This is because some of these herbs can be injurious to health, including death, due to intoxication or poisoning.

This is probably one of the reasons for high mortality rate attributable to mothers and babies during delivery. The overall implications is that TBAs need more education on the use of drugs and herbs as most of them use herbs singly or in combination with orthodox medicine as found in this study.

Another point is that most of TBAs are illiterate, no matter how effective their local herbs might be, it is important to remember that the active ingredients are not known and the dosage cannot be quantified. It is necessary therefore for further studies to be carried out to determine the content or the active ingredients of these herbs and their efficacies. The dosages also need to be determined as well. This is because nobody knows what harmful effects they may be inflicting on the body which may surface in one way or the other. On the other hand, these herbs may be considered good when analysed and could lead to a major breakthrough in medicine as most drugs use in treatment are extracted from herbs [8].

The finding that TBAs refer mothers to hospital when their maneuver during complications has failed is a major and common phenomenon, except few that said they don't touch immediately they notice danger. Hence there is need to educate the TBAs on the early signs of danger or risk and the need for prompt referral of patients to qualified health practitioners before it is late. This could save the mother and newborn life and lead to reduction of maternal and infant mortality. This is in line with the report of, [1].which indicates that TBAs can potentially improve maternal and new born health at community level. Nevertheless, while the role of TBAs in caring for pregnant women and conducting deliveries is acknowledged, it is noted that they are generally not trained to deal with complications,[2].

\section{Conclusion}

TBAs have been unsuccessful in handling obstetric complications, but have contributed to successful maternal, neonatal, and child health interventions. The key piece missing in TBA training is an adequate referral system, which allows TBAs to use their close ties with the community to link pregnant women to skilled birth attendants (SBA). The effectiveness of TBA referrals to health facilities is dependent on the healthcare system's ability to support an increase in services provided and the supervision needed to support TBA training and integration

\section{Reference}

[1]. World Health Organization.Global Action for Skilled Attendants for Pregnant Women. Geneva: WHO 2004

[2]. World Health Organization (WHO) World Health Report 2005: Make Every Mother and Child Count. Geneva: WHO; 2005

[3]. S.M Itina SM. Characteristics of traditional birth attendants and their belief and practices in the Ofot clan, Nigeria. Bull World Health Organ. 1997;75(6):537-538.[PMC free article] [PubMed

[4]. Itina SM. Characteristics of traditional birth attendants and their belief and practices in the Ofot clan, Nigeria. Bull World Health Organ. 1997;75(6):537-538.[PMC free article] [PubMed]

[5]. A.O, Imogie, Agwubike EO, Aluko K. Assessing the role of traditional birth attendants (TBAs) in health care delivery in Edo State,

[6]. Nigeria. Afr J Reprod Health. 2002;6(2):94-100. [PuHYPERLINK "http://www.ncbi.nlm.nih.gov/pubmed/12476721"bMed]

[7]. A.NOfili, and Okojie OH. Assessment of the role of traditional birth attendants in maternal health care in Oredo Local Government Area, Edo State, Nigeria. Journal of Community Medicine and Primary Health Care. 2005;17(1):55-60.

[8]. K. Peltzer, Henda N. Traditional birth attendants, HIV/AIDS and safe delivery in the Eastern Cape, South Africa - evaluation of a trainingprogramme. S Afr J ObstetGynaecol. 2006;12(3):140-145.

[9]. O.AAhmed, Odunukwe NN, Akinwale OP, et al. (2005) Knowledge and Practices of Traditional Birth Attendants in Prenatal Services in Lagos State, Nigeria.Afr J Med Sci 